\title{
Study on Reliability of Distribution System with Unreliable Distributed Generation
}

\author{
Ping Wang ${ }^{1, a}$, Zhaoyuan Liu ${ }^{1, b}$ \\ ${ }^{1}$ State Grid Qingdao Power Supply Company, Qingdao Shandong, PRC \\ aqdwangping73@163.com, b1062353207@qq.com
}

\begin{abstract}
Keywords: distributed generation, reliability evaluation, failure delivery, interval arithmetic, island. Abstract. Increasing insertion of distributed generations(DGs) challenges power grid security. Based on analysis of failure impact delivery which is divided into the counter-flow delivery and the direct-flow delivery, the approach of distribution system reliability evaluation is proposed. Because of no accurate value of some data in practice, interval arithmetic is introduced to calculate reliability indices. On the basis of analysis of output instability of DG, recovery probability index is used to be on behalf of the probability of island operation. Then distribution system reliability indices are calculated with this method. Case calculation of a $10 \mathrm{kV}$ feeder at Qingdao verifies improvement of system reliability with the insertion of DG, show that access point and island partition that play an important role in reliability of loads must be considered in scientific planning of insertion of DG.
\end{abstract}

\section{Introduction}

As an efficient utilizing way of renewable energy, more distributed generations (DGs) have been inserted into power grid which impacts the topology and operation of distribution system. Distribution system plays a key role in customer service reliability. Unreliable DG with the random power fluctuation characteristic changes power flow of distribution network and brings on uncertainties in the stable operation of power grid.

Traditional reliability analysis methods of distribution network, such as Failure modes and effects analysis(FMEA), the minimal-path method, the reliability-network-equivalent and Monte Carlo simulation, can not be used in reliability analysis of distribution system with unreliable DG. [1] proposes an exponential smoothing method to building an accurate multi-state output model of wind turbine generators. [2] deals with power fluctuation of DG and load based on point estimation method and then implements the point estimation of reliability indices according to the relationship between the above two and the reliability of distribution network. [3] obtains output of wind turbine according to the probability distribution of wind speed in one year and accesses reliability by improved minimal cut set algorithm. [4] divides islands in the principle of power balance in maximum load and on the basis of simulation, the problem of reliability calculation when probability models of DG included is approached. [5] construct the supply region of wind power and calculates the probability of islanding operation. [6] simplifies distribution network by feeder partition method and considering the probability models of DG and load, authors propose a fast reliability evaluation method. [7] divides islands according to the economic model and improved minimal path method is used to obtain the reliability indices when considering adverse weather. [8] builds a multi-state output model of resources based on the Markov method and deals with the problem of power supply probability with bi-state output power model.

The effects of insertion of unreliable DG in distribution system are studied in this paper which begins from reliability evaluation method. Firstly based on the relationship of components in grid faults, the failure impact delivery is divided into the counter-flow delivery and the direct-flow delivery. Secondly failure impacts on loads in island and loads not in island are respectively analyzed on the basis of supply recovery probability. And then calculate reliability indices by improved minimal-path method. Finally by taking a $10 \mathrm{kV}$ feeder at Qingdao as an example, impacts of DG on distribution network are analyzed. 


\section{Failure impact delivery}

There exist interactions among components of distribution network. In a simple network as shown in Fig.1, when L3 breaks down, relay protection makes circuit breakers B3 and B4 operating and with the operation of B4, power of the adjacent component L4 is cut off. Failure impact keeps on being delivered until it is at the terminal of power circuits.

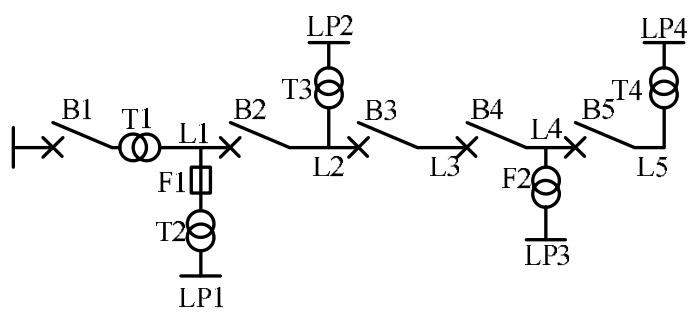

Fig.1. The diagram of simple distribution network

On the basis of above analysis, it can be generalized that failure impact is delivered from one component to adjacent components and reduces reliability of components and loads.

The counter-flow delivery. Following cases are considered:

1) Failure is delivered from a fuse or breaker. If the fuse/breaker is reliable, there is no influence on upstream component until the fuse or breaker fails to operate.

2) Failure is delivered from non-switch components like lines, transformers and so on. Failure is impervious to the isolation of these components.

3) A component can be isolated by isolating switches. Then the outage frequency caused by this component is invariable but the interruption time turns to the isolation time of failure.

Define $\lambda^{\mathrm{u}}{ }_{\mathrm{i}}$ as the number of power cut in one year in a counter flow delivery from the component $\mathrm{i}$; $U^{\mathrm{u}}{ }_{\mathrm{i}}$ is the interruption time in one year in a counter flow delivery from the component $\mathrm{i}$; the component $\mathrm{j}$ is the adjacent upstream component of the component $\mathrm{i}$.

(1) if $\mathrm{j}$ is connected to unique downstream branch circuit, then

$\lambda_{\mathrm{j}}^{\mathrm{u}}=\left\{\begin{array}{c}p_{\mathrm{j}} \cdot \lambda_{\mathrm{i}}^{\mathrm{u}}+\lambda_{\mathrm{j}}, \mathrm{j} \text { is a breaker or fuse } \\ \lambda_{\mathrm{i}}^{\mathrm{u}}+\lambda_{\mathrm{j}}, \mathrm{j} \text { is a non - switch component }\end{array}\right.$

$U_{\mathrm{j}}^{\mathrm{u}}=\left\{\begin{array}{c}p_{\mathrm{j}} U_{\mathrm{i}}^{\mathrm{u}}+\lambda_{\mathrm{j}} T_{\mathrm{j}}, \mathrm{j} \text { is a breaker or fuse can be isolated separately } \\ p_{\mathrm{j}} U_{\mathrm{i}}^{\mathrm{u}}+U_{\mathrm{j}}, \mathrm{j} \text { is a breaker or fuse can't be isolated separately } \\ U_{\mathrm{i}}^{\mathrm{u}}+U_{\mathrm{j}}, \mathrm{j} \text { is a non - switch component }\end{array}\right.$

$p_{\mathrm{j}}$ is the unreliable rate of the component $\mathrm{j} ; \lambda_{\mathrm{j}}$ is the failure rate of the component $\mathrm{j} ; U_{\mathrm{j}}$ is the interruption time in a year of the component $\mathrm{j} ; T_{\mathrm{j}}$ is the isolation time of failure of the component $\mathrm{j}$.

(2) if $\mathrm{j}$ is connected to several downstream branch circuits, then

$\left\{\begin{array}{c}\lambda^{\mathrm{u}}{ }_{\mathrm{j}}=p_{\mathrm{j}} \sum_{\mathrm{k} \in N_{\mathrm{j}}} \lambda_{\mathrm{k}}^{\mathrm{u}}+\lambda_{\mathrm{j}} \\ U^{\mathrm{u}}{ }_{\mathrm{j}}=p_{\mathrm{j}} \sum_{\mathrm{k} \in N_{\mathrm{j}}} U_{\mathrm{k}}^{\mathrm{u}}+e U_{\mathrm{j}}+(1-e) \lambda_{\mathrm{j}} T_{\mathrm{j}}\end{array}\right.$

$e=\left\{\begin{array}{l}0, \mathrm{j} \text { can be isolated separately } \\ 1, \mathrm{j} \text { can't be isolated separately }\end{array}\right.$

$N_{\mathrm{j}}$ is the collection of adjacent downstream components of the component $\mathrm{j}$. While the component $\mathrm{j}$ is a non-switch component, $p_{\mathrm{j}}$ viewed as the unreliable rate of the component $\mathrm{j}$ is $100 \%$.

The direct-flow delivery. the direct-flow delivery is different. The fault in upstream will surely lead to the outage in downstream components as a result of the direct power flow.

Define $\lambda_{i}^{\mathrm{d}}$ as the number of power cut in one year in the direct-flow delivery from the component $i$; $U^{\mathrm{d}}{ }_{\mathrm{i}}$ is the interruption time in one year in the direct-flow delivery from the component $\mathrm{i}$; the component $\mathrm{j}$ is the adjacent downstream component of the component $\mathrm{i}$.

$$
\left\{\begin{array}{c}
\lambda_{\mathrm{j}}^{\mathrm{d}}=\lambda_{\mathrm{i}}^{\mathrm{d}}+\lambda_{\mathrm{j}} \\
U_{\mathrm{j}}^{\mathrm{d}}=U_{\mathrm{i}}^{\mathrm{d}}+U_{\mathrm{j}}
\end{array}\right.
$$




\section{Calculating reliability indices of distribution network}

Network simplification base on feeder partition method. Based on feeder partition method ${ }^{[9]}$, the connected region isolated by isolation devices is defined as the feeder zone. So the whole distribution network consist of feeder zones and the connection points are switching components such as circuit breakers and fuses. The network in fig. 1 is simplified as shown in fig. 2.

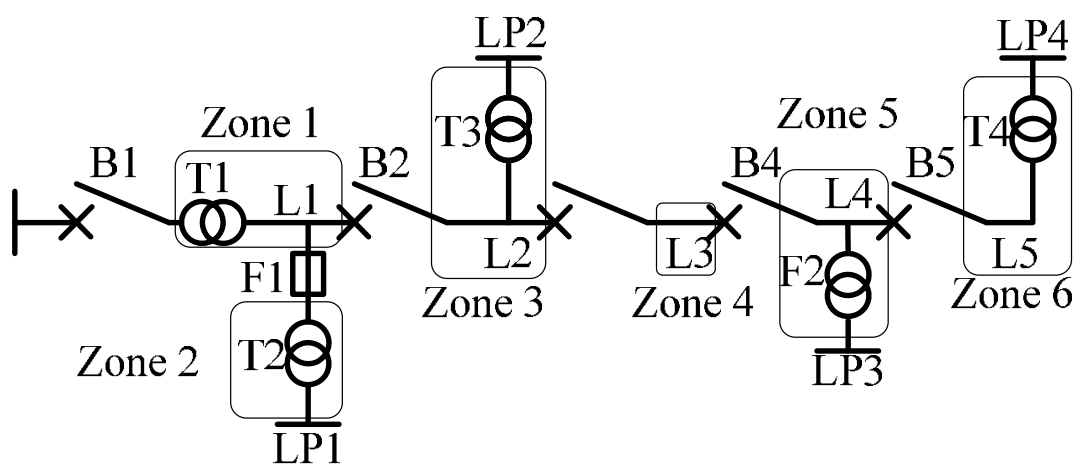

Fig.2. Feeder partition

It is as a result of no switch components inside that a feeder zone is viewed as a non-switch component in power system reliability evaluation.

The reliability parameters of the whole zone are calculated as follows:

$$
\left\{\begin{array}{l}
\lambda_{\mathrm{i}}=\sum_{k \in S_{\mathrm{i}}} \lambda_{k} \\
U_{\mathrm{i}}=\sum_{k \in S_{\mathrm{i}}} U_{k}
\end{array}\right.
$$

$S_{\mathrm{i}}$ is the collection of components in the feeder zone $\mathrm{i}$.

Interval arithmetic. In the practice, much data can not be got accurately or the original data itself is an interval value. For example, the failure rate of a component in distribution network, influenced by surroundings and its state, is a value changing with the time. The output of DG is also like that. In such circumstances, not an exact value but an interval value is an relative precision to describe the real performance ${ }^{[10]}$.

If $\bar{x}$ and $\underline{x} \in \mathrm{R}$ ( $\mathrm{R}$ is the real field) and $\underline{x} \leq \bar{x}$, then the closed bounded number set is

$$
X=[\underline{x}, \bar{x}]=\{x \in \mathrm{R} \mid \underline{x} \leq x \leq \bar{x}\}
$$

$\underline{x}$ is the lower limit of the interval number $X ; \bar{x}$ is the upper limit of the interval number $X$.

While $\mathrm{x}=\bar{x}$, define the interval number $[\underline{x}, \bar{x}]$ as the point interval number. For example, the constant 1 is viewed as the interval number $[1,1]^{[11]}$.

If the interval numbers $X=[\underline{x}, \bar{x}]$ and $Y=[y, \bar{y}]$, there are four fundamental operations as follows:

$$
\begin{aligned}
& X+Y=[\underline{x}+\underline{y}, \bar{x}+\bar{y}] \\
& X-Y=[\underline{x}+\bar{y}, \bar{x}-\underline{y}] \\
& X Y=[\min (\underline{x} \underline{y}, \underline{x} \bar{y}, \bar{x} \underline{y}, \overline{x y}), \max (\underline{x} \underline{y}, \underline{x} \bar{y}, \bar{x} \underline{y}, \overline{x y})] \\
& X / Y=[\underline{x}, \bar{x}] \cdot[1 / \bar{y}, 1 / \underline{y}] \quad(\text { If } Y \neq 0)
\end{aligned}
$$

Calculation of reliability indices based on improved minimal path method. On the basis of minimal path, network is divided into minimal path and its branches when calculate reliability indices of a load point in a distribution network. Define the switch component joining the minimal path and a branch as the branch first point. Calculate reliability indices of a load point with the direct flow from the power source to the load point in the minimal path and the counter flow from the end point to the first point in branch circuits.

$$
\left\{\begin{array}{c}
\lambda=\lambda^{u}+\lambda^{d} \\
U=U^{u}+U^{d}
\end{array}\right.
$$

In contrast to above calculation without the power supply of DG, the influence of islanding operation must be considered in distribution network with unreliable DG. 
If the load point $\mathrm{i}$ is included in an island, the load point $\mathrm{i}$ can be powered by DG in probability as long as the network from DG to the load point is connective when upstream network fails. Then the interruption duration turns to be the load transfer duration.

While there is a power supply path from DG to a load point which is defined as the minimal path in island, the minimal path from power source to the load point is defined as the minimal path in power network. Above two paths are intersect at a feeder zone called $\mathrm{C}_{\mathrm{i}}$. So the reliability index of a load point consists of two main parts-upstream failure of $C_{i}$ and failure in the path from $C_{i}$ to the load point.

Considering unreliable DG with the random power fluctuation characteristic, there is probability of DG supply. This paper argues that the recovery probability supplied by DG should be obtained by statistic analysis. the recovery probability is defined as the ratio of reduced failure time, owing to power supply by DG, to the original failure time when failure, can be recovered by DG, happens. The recovery probability $P_{\mathrm{r}}$ is expressed by the following equation:

$$
P_{r}=1-\frac{\sum \gamma_{r i}}{\sum \gamma_{i}}
$$

$\Sigma \gamma_{\mathrm{ri}}$ is the interruption time in the failure recovered by DG and $\Sigma \gamma_{\mathrm{i}}$ is the corresponding repair time.

On the basis of above study, reliability indices of a load point in distribution network with unreliable DG are expressed by the following equations:

$$
\left\{\begin{array}{c}
\lambda_{\mathrm{LPi}}=\lambda^{\mathrm{d}}+\sum_{\mathrm{k} \in S^{\mathrm{bi}}} \lambda_{\mathrm{k}}^{\mathrm{u}} \\
U_{\mathrm{LPi}}=\left(1-P_{\mathrm{r}-\mathrm{i}}\right)\left(U_{\mathrm{s}-\mathrm{ci}}^{\mathrm{d}}+\sum_{\mathrm{k} \in S_{\mathrm{s}-\mathrm{i}}^{\mathrm{u}}} U_{\mathrm{k}}^{\mathrm{u}}\right) \\
+\left(U_{\mathrm{ci}-\mathrm{i}}^{\mathrm{d}}+\sum_{\mathrm{k} \in S_{\mathrm{ci}-\mathrm{i}}^{\mathrm{b}}} U_{\mathrm{k}}^{\mathrm{u}}\right)
\end{array}\right.
$$

$\lambda_{\mathrm{LPi}}$ and $U_{\mathrm{LPi}}$ are the number of power cut in one year of the load point $i$ and the interruption time in one year of the load point i respectively; $P_{\mathrm{r}-\mathrm{i}}$ is the recovery probability of the load point i. $S^{\mathrm{bi}}$ is the collection of branch first points; $\mathrm{Sbi} \mathrm{S}-\mathrm{Ci}$ is the collection of branch first points corresponding to the minimal path from power source $\mathrm{S}$ to the feeder zone $\mathrm{C}_{\mathrm{i}}$; $\mathrm{Sbi} \mathrm{Ci}-\mathrm{i}$ is the collection of branch first points corresponding to the minimal path from feeder zone $C_{i}$ to the load point $i . \lambda^{\mathrm{d}}$ means the number of power cut in the direct-flow delivery. $U \mathrm{~d} \mathrm{~S}-\mathrm{Ci}$ is the interruption time delivered from the component in front of feeder zone $\mathrm{C}_{\mathrm{i}}$ in the direct-flow delivery; $U \mathrm{~d} \mathrm{Ci}-\mathrm{i}$ is the interruption time of the path from feeder zone $\mathrm{C}_{\mathrm{i}}$ to the component in front of the load point $\mathrm{i}$.

\section{Case studies}

The system under study is a simplified 10kV feeder at Qingdao as shown in fig. 3 .

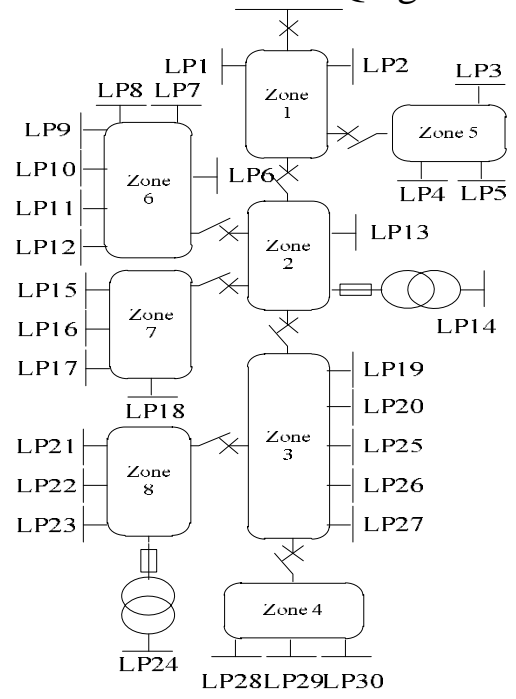

Fig.3. Example feeder 
In this case, the unreliability probability, failure rate and repair time of circuit breakers are $10 \%$, 0.02 times per year and 2 hours each time respectively while their interval values are [10\%, 10\%], $[0.018,0.022]$ and $[1.6,2.4]$ respectively; the failure rate and repair time of transformers are 0.015 times per year and 10 hours each time respectively while their interval values are $[0.014,0.016]$ and $[8$, 12]; the reliability probability of fuses is $100 \%$ and their failure is ignored; all circuit breakers can be isolated separately by isolating switches and the isolation time is 1 hour while its interval value is [0.7, 1.3]; the repair time of circuits is 4 hours while its interval value is [3.2, 4.8]. The indices of each zone are shown in Table 1.

There are 3 plans analyzed with a 2MW DG just shown in Table 2.Because of the small impact on reliability of loads not in island, here is comparisons between reliability indices of these plans in the respective islands. The results of these calculations are shown in Table 3 and Table 4.

Table 1. Indices of each zone

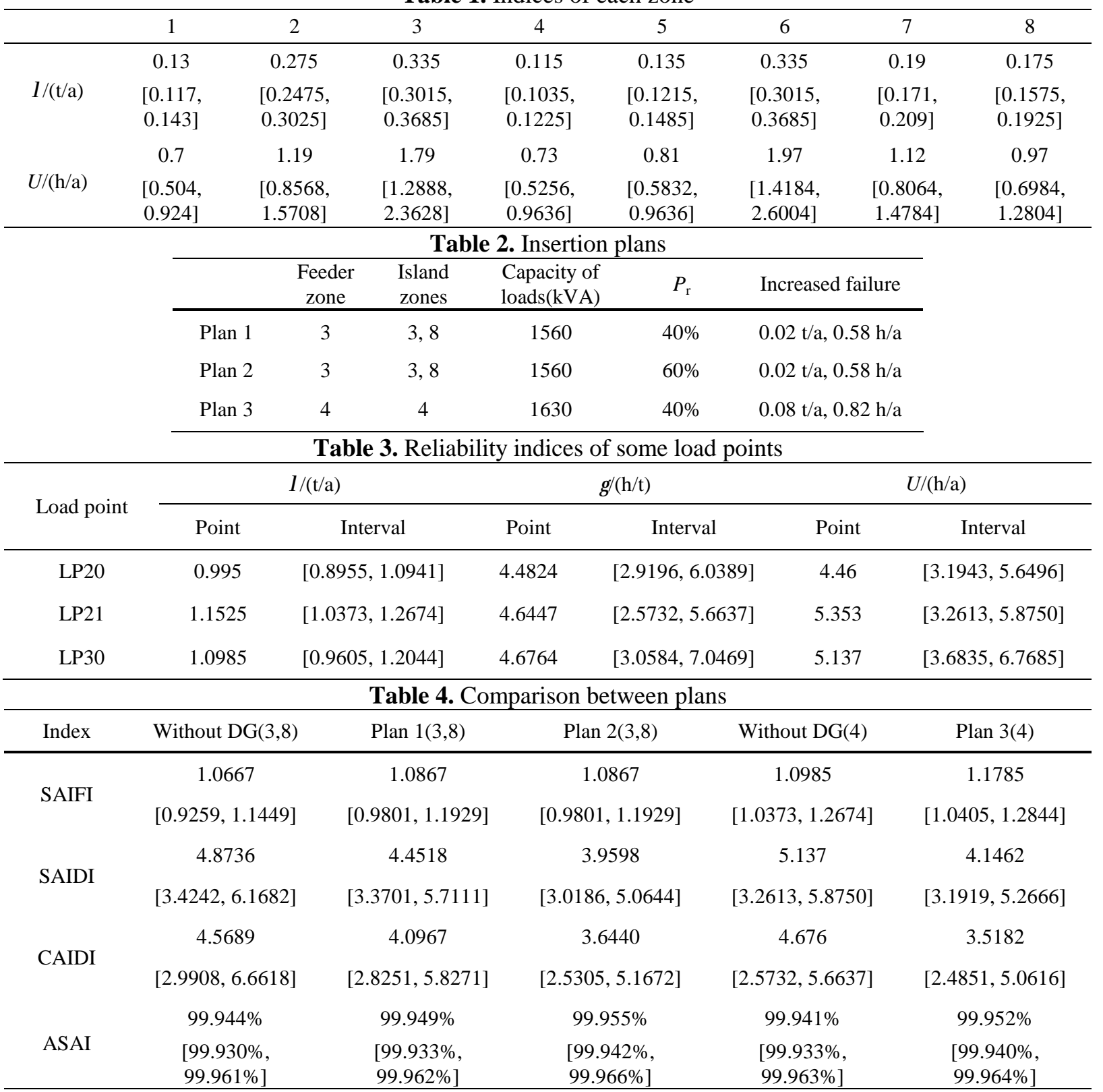

The recovery probability, turning to $60 \%$ from $40 \%$, is the only difference between Plan 1 and Plan 2. For the improvement of reliability, the interruption time, through island reduces it, is not improved in the same level to the increase of the recovery probability. On the basis of analysis on calculation, the main reason is that the insertion of DG reduces reliability of the feeder zone connected at the same time. So if failures in upstream are not so frequent, power supply reliability can not be improved much despite a high recovery probability. 
Calculation shows that the insertion of DG makes little influence on the number of power cut if load transfer is accounted. The reason is that when a failure happens, there is an island operation after a period of time due to the fault location and energy management in island. The insertion of DG complicates protection strategy in network.Connecting to network at the access point near to the end line can reduce interruption time effectively. Above all, the optimal insertion of DG depends on actual conditions such as island operation, the type of load and the characteristics of DG.

\section{Conclusions}

In summary, the insertion of DG does improve power supply reliability but the improvement will vary according to islanding operation. There are 3 main suggestions:

1) Not only the access point nearby but also the access point not too far must be taken to account. Perhaps the access point not too far may improve reliability and reduce loss more effectively with upstream failures and loads considered.

2) Improve the probability of the recovery probability. Increase power supply margin of DG in island on the one hand and enhance the ability of stable output of DG on the other hand.

3) Both of the capacity of loads in island and the access point should be considered in island partition so that more customers are powered and powered stably.

\section{Acknowledgments}

This work was financially supported by Science and Technology Project of State Grid Shandong Electric Power Company.

\section{References}

[1] X.W. Qi, K.G. Xie and B. Hu: Power Sys. Prot. Contr. Vol. 40(No.12 2012), p. 1(In Chinese)

[2] J.J. Lu, Y. Zhao, Y.S. Zhao and K.G. Xie: Power Sys. Technol. Vol. 37(2013), p. 2250(In Chinese)

[3] Y.Q. Xu and Y.C. Wu: Power Sys. Technol. Vol. 35(No.4 2011), p. 154(In Chinese)

[4] Z. Lei, G. Wei, Y. Cai and X. Zhang: Auto. Elec. Power Sys. Vol. 35(No.1 2011), p. 39(In Chinese)

[5] Y.Q. He, J.C. Peng and Q. Sun: Proc. CSEE Vol. 30(No.13 2010), p. 16(In Chinese)

[6] Z.K. Li, L.J. Wang, G. Wang, Z.L. Yang and Y. Zhang: Auto. Elec. Power Sys. Vol. 37(No.4 2013), p. 35(In Chinese)

[7] Y. Li, J.Y. Liu, Z.B.Wei, Y.B. Liu, Z.T. Xu and R. Zhang: Power Sys. Prot. Contr. Vol. 40(No.2 2012), p. 93(In Chinese)

[8] S. Wang, W. Tan and H. Huang: Elec. Power Auto. Equip. Vol. 35(No.4 2015), p. 31(In Chinese)

[9] Y.H. Xie and C.S. Wang: Proc. CSEE Vol. 24(No.5 2004), p. 35(In Chinese)

[10] K.J. Qian, Y. Yuan and C.K. Zhou: Power Sys. Technol Vol. 32(No.11 2008), p. 74(In Chinese)

[11]P. Zhang and S.X. Wang: Proc. CSEE Vol. 24(No.3 2004), p. 77(In Chinese) 